Research Paper

\title{
Autophagy Inhibition Stimulates Apoptosis in Oesophageal Squamous Cell Carcinoma Treated with Fasudil
}

\author{
Fa-Jun Xie ${ }^{1,2 \llbracket *}$, Qiu-Qing Zheng ${ }^{2,3^{*}}$, Jing Qin ${ }^{1,2}$, Ling-Ling Zhang ${ }^{4}$, Na Han ${ }^{1,2}$, Wei-Min Mao ${ }^{2,5 \bowtie}$ \\ 1. Department of Medical Oncology, Zhejiang Cancer Hospital, Hangzhou 310022, China. \\ 2. Zhejiang Key Laboratory of Diagnosis and Treatment Technology on Thoracic Oncology (Lung and Oesophagus), Hangzhou 310022, China. \\ 3. Department of Ultrasonography, Zhejiang Cancer Hospital, Hangzhou 310022, China. \\ 4. Department of Medical Oncology, Peking University International Hospital, Beijing 102206, China. \\ 5. Department of Thoracic Surgery, Zhejiang Cancer Hospital, Hangzhou 310022, China. \\ "These authors contributed equally to this work.
}

$\triangle$ Corresponding author: Doctor Fa-Jun Xie, Department of Medical Oncology, Zhejiang Cancer Hospital, No. 1 East Banshan Road, 310022, Hangzhou, Zhejiang Province, China. Phone: +86 571-88122091; Fax: +86 571-88122091; E-mail addresses: xiefj@zjcc.org.cn or Wei-Min Mao, Professor, Zhejiang Key Laboratory of Diagnosis and Treatment Technology on Thoracic Oncology (Lung and Oesophagus), No. 1 East Banshan Road, Hangzhou 310022, Zhejiang Province, China. E-mail addresses: maowm1218@163.com.

(c) Ivyspring International Publisher. This is an open access article distributed under the terms of the Creative Commons Attribution (CC BY-NC) license (https://creativecommons.org/licenses/by-nc/4.0/). See http://ivyspring.com/terms for full terms and conditions.

Received: 2017.10.18; Accepted: 2018.01.28; Published: 2018.02.28

\begin{abstract}
Fasudil has been proven to be a promising chemotherapeutic drug for various malignancies. However, the potential anticancer effects of fasudil in oesophageal squamous cell carcinoma (ESCC) remain to be established. We confirmed the RhoA activity is inhibited by fasudil in ESCC cells. Then measured the effects of fasudil on apoptosis and autophagy in ESCC. Our study showed fasudil could both induce ESCCs apoptosis and autophagy, and when fasudil-induced autophagy was inhibited by knockdown of the essential autophagy genes (Beclin 1 or ATG7), and pharmacologic agent (chloroquine) treatment, both treatments also significantly sensitized ESCC to fasudil-induced apoptosis, reducing cell viability in vitro. Our study showed autophagy inhibitors combined with fasudil could significantly induce ESCC apoptosis, which may provide a novel therapeutic strategy for ESCC.
\end{abstract}

Key words: Fasudil, Oesophageal squamous cell carcinoma, Apoptosis, Autophagy

\section{Introduction}

Oesophageal squamous cell carcinoma (ESCC) is the domination histological subtype of oesophageal cancer, accounting for more than $90 \%$ of this malignancy [1]. The 5-year overall survival (OS) rate of patients with ESCC is less than $10 \%$ [2]. Its unfavourable prognosis is mainly explained by the fact that patients are usually diagnosed at an advanced stage. Even in operable patients, more than $50 \%$ suffer local recurrence or distant metastasis [3]. Chemotherapy is the mainstay of palliative treatment for advanced or recurrent cancer; however, its poor outcomes and severe toxicity urgently require that novel drugs be explored.
The RhoA/ROCK pathway plays a critical role in ESCC progression, including promoting tumour cell growth, migration, metastasis and poor prognosis $[4,5]$. RhoA/Rock signal pathway is as recognized as one of the upstream regulators of Beclin-1-mediated autophagy and regulates the homeostasis between autophagy and apoptosis[6]. Indeed, adenovirusmediated RhoA shRNA suppresses the growth of ESCC cells in vitro and in vivo [7]. However, the lack of efficacy and accuracy of virus vectors infecting the tumour cells restrict the practical application of adenovirus-mediated gene therapy for cancer[8].

Fasudil, a pharmacological RhoA/ROCK pathway inhibitor, is a clinically available drug without 
obvious adverse effects on cardiovascular disease[9]. In addition, fasudil inhibits tumour growth, metastasis, and invasion in a variety of tumour types, including fibrosarcoma [10], hepatocellular carcinoma [11], acute myeloid leukaemia [12] and lung cancer [13]. It also plays a role in anti-angiogenesis in tumours by inducing hypoxia-inducible factor-1alpha degradation [14]. However, the potential anti-cancer effects of fasudil in ESCC have not been fully characterized.

In this study, we aimed to show that the RhoA inhibitor fasudil induces ESCC autophagy and apoptosis. By employing pharmacologic and genetic agents to inhibit autophagy, the proapoptotic effect induced by fasudil was enhanced significantly. These results suggest that fasudil combined with an autophagy inhibitor is a novel and promising strategy for the treatment of ESCC.

\section{Materials and methods}

\section{Cell culture and reagents}

Oesophageal squamous cell lines EC109 and KYSE150 were maintained by our laboratory and cultured in DMEM (Gibco, Grand Island, NY, USA) supplemented with 10\% FBS (HyClone, Logan, UT, USA), penicillin $(100 \mathrm{U} / \mathrm{mL})$ and streptomycin $(100$ $\mathrm{mg} / \mathrm{mL}$ ) (Gibco). GAPDH, microtubule-associated protein 1 light chain 3 (LC3), Beclin1, caspase 3, and caspase 9 antibodies were ordered from Cell Signaling (Cell Signaling Technology, Boston, MA, USA). The secondary antibodies HRP-conjugated anti-rabbit and anti-mouse IgG were purchased form Cell Signaling Technology. Fasudil (Tianjin Chase Sun Pharmaceutical Co., Ltd., Tianjin, China) was diluted in PBS.

For evaluating fasudil-induced apoptosis, ESCC cells were sub-cultured on a 24 -well plate covered with $13 \mathrm{~mm} \times 13 \mathrm{~mm}$ cover slips and exposed to fasudil. The cells were fixed with $10 \%$ absolute methanol, permeabilized with $0.25 \%$ Triton X-100 and stained with DAPI $(1 \mathrm{~g} / \mathrm{ml})$ for $10 \mathrm{~min}$. The morphologic changes in characteristic apoptotic nuclei were examined by confocal microscopy (Olympus, Tokyo, Japan).

\section{DAPI nuclear staining assay}

Cells were cultured onto slides in six-well plate and exposed to fasudil for indicated times, then collected and fixed in $0.1 \%$ paraform for $30 \mathrm{~min}$. After being washed in PBS, the slides were soaked in DAPI staining solution $(100 \mu \mathrm{g}$ DAPI dissolved in $100 \mathrm{~mL}$ PBS that contain $0.1 \%$ TritionX-100) for $10 \mathrm{~min}$ in the dark. The morphologic changes of apoptosis characteristic nuclei were examined by confocal microscopy (Olympus).

\section{Cell inhibition Assay}

Cells were growth on a 96-well plate (Falcon; BD Biosciences Discovery Labware, Bedford, MA) at a cell density of 4000 per well, and diverse concentrations of compounds were added to the culture medium and incubated for the indicated time. Cell proliferation was detected by the CCK- 8 assay as reported previously [15].

\section{Foci formation assay}

Cells (1000 cells/2 ml/well) were planted in six-well plates and were deal with fasudil at diverse concentrations $(0-100 \mathrm{nM})$ for $48 \mathrm{~h}$. After being washed with fresh medium, the cells were incubated to grow for 21 days to form cell colonies, which were then stained with $1 \%$ crystal violet, and the colonies containing more than 50 cells were counted.

\section{Annexin V/PI staining}

Both floating and adherent cells were harvested after fasudil treatment $(48 \mathrm{~h})$ and were rinsed with $4^{\circ} \mathrm{C}$ phosphate-buffered saline (PBS). Cells were then resuspended in $5 \mathrm{ml}$ of Annexin V and $5 \mu \mathrm{PI}$ and incubated for $30 \mathrm{~min}$ at room temperature in the dark (FITC Annexin V Apoptosis Detection Kit I, BD Bioscience, Bedford, MA, USA). Cell survival was evaluated by flow cytometric analysis using a FACS Calibur flow cytometer (Becton Dickinson, San Jose, CA, USA).

\section{RNA interference}

Knockdown of Beclin 1 gene expression was obtained by transfection of small interfering RNA (siRNA), which was purchased from Ambion (Norwalk, CT, USA). A negative control siRNA and Oligonucleotide duplexes targeting Beclin 1 (s16537) were ordered from Ambion (Norwalk, USA). ATG7 siRNA was purchased from Qiagen company (SI02655373); The siRNA was transfected into the cells using FuGENE HD Transfection Reagent (Promega, USA) in accordance with the manufacturer's instructions.

\section{RhoA activation assay and Western Blot Analysis}

To detect the phosphorylated active RhoA, we used a Rho-binding domain (RBD) affinity precipitation assay for RhoA-GTP with a specific antibody targeting RhoA (Cytoskeleton Inc., Denver, CO, USA).

For western blot analysis, the cells were collected and lysed in $1 \times$ cell lysis buffer (Cell Signalling Technology) with the addition of $1 \mathrm{mM}$ phenylmethanesulfonylfluoride fluoride immediately before employ. Equal amounts of protein extract $(10 \sim 30 \mu \mathrm{g})$ 
were preformed to $10 \%$ sodium dodecyl sulfatepolyacrylamide gel electrophoresis (SDS-PAGE) and blotted onto polyvinylidene fluoride membranes (Millipore, Belford, MA). After blocking, the membranes were hatched with primary antibodies for LC3-I/II, caspase-3, caspase-9 and GAPDH. Blots were then hatched with a horseradish peroxidase (HRP)-conjugated anti-mouse or anti-rabbit antibody. Signals were detected via enhanced chemiluminescence using Immobilon Western HRP Substrate (Millipore, Billerica, MA).

\section{Caspase activity assay}

Cells were seeded in $175-\mathrm{cm}^{2}$ flasks. After hatch with $50 \mu \mathrm{M}$ fasudil for $48 \mathrm{~h}$, floating and adherent cells were collected and merged. Preparation of cell lysates and the evaluation of caspase- 9 and caspase- 3 activity was performed with a Caspase-9 and Caspase-3 Cellular Activity Assay Kit (Calbiochem,
La Jolla, CA, USA) according to the manufacturer's instructions.

\section{Statistical analysis}

Results are shown as the mean \pm standard error $(\mathrm{SE})$. Statistical significance $(\mathrm{P}<0.05)$ was determined by Student's t-test or analysis of variance (ANOVA), followed by an assessment of differences applying IBM SPSS 19.0 software (SPSS Inc., Chicago, IL, USA).

\section{Results}

\section{RhoA activation in ESCC cells was inhibited by fasudil treatment}

To detect the effect of fasudil on RhoA activation in human ESCC cells, we treated EC109 and KYSE150 cells with 0-100 $\mathrm{nM}$ fasudil for $24 \mathrm{~h}$ and measured the Rho activation by small G-protein activation assay. We found that fasudil had a potent inhibitory effect on RhoA activation (Fig. 1A).

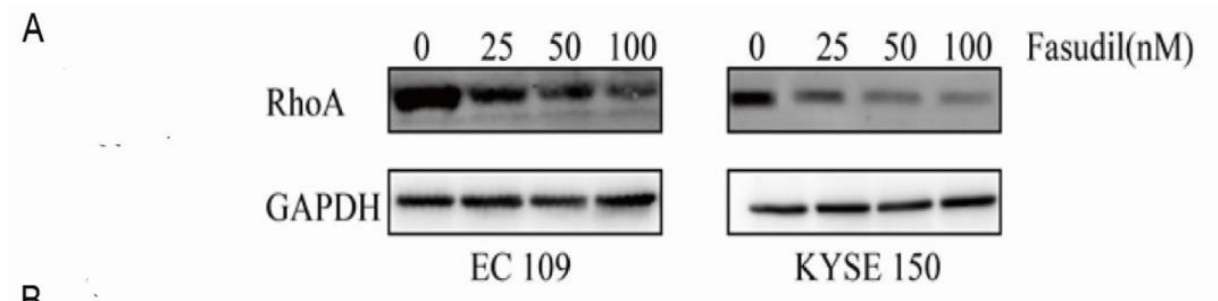

B

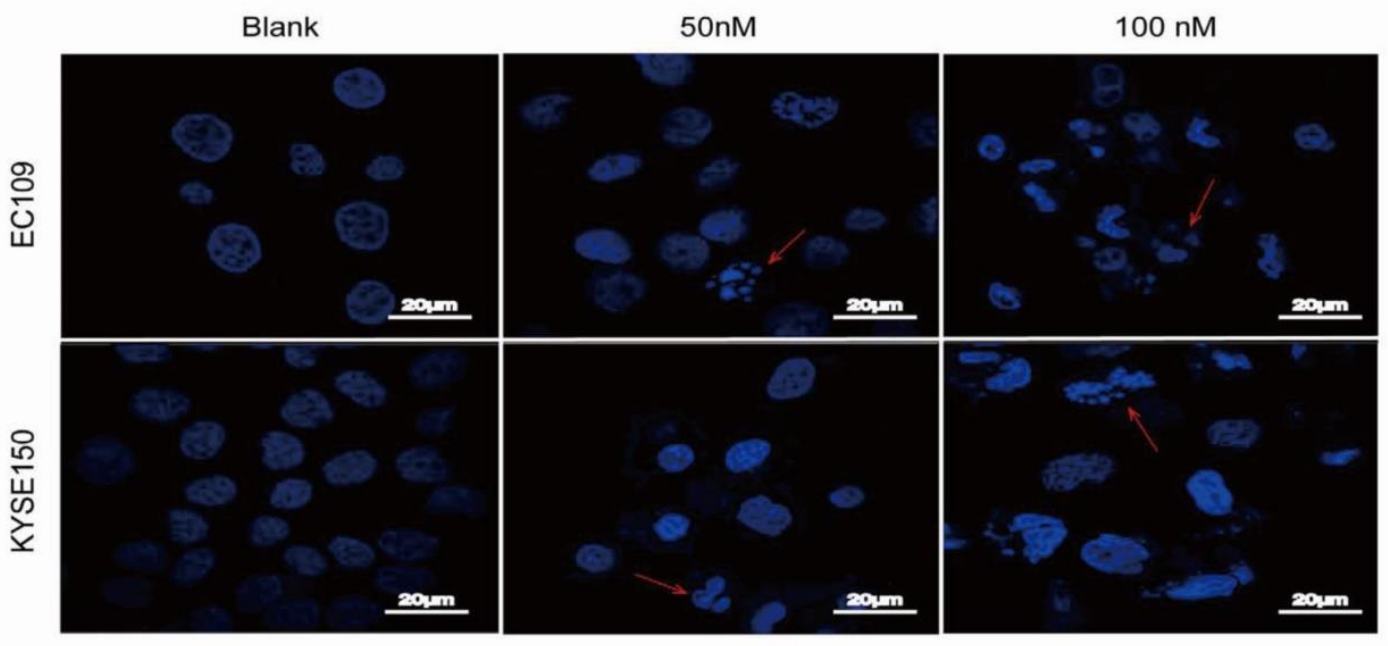

C $0255075(\mathrm{nM})$

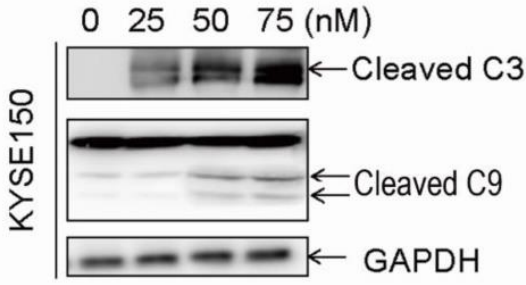

Figure 1. RhoA inhibitor induced ESCCs apoptosis. (A)Inhibitory effects of fasudil on the RhoA pathway in EC109 and KYSE150 cells. Cells were seeded in a 6-well plate; when the cell growth reached approximately $80 \%$ intensity, diverse concentrations of fasudil were added to the culture medium and incubated for 24 h. The activity of RhoA was detected by RhoA activation assay. The results are representative of three different experiments. (B)Characteristic apoptosis was seen in KYSE150 and EC109 cells treated with fasudil for $48 \mathrm{~h}$. (Original magnification, 200x). (C) Western blot analysis was performed with cleaved caspase-3 antibody and caspase-9 antibody probes. Blank: PBS treatment. GAPDH, glyceraldehyde-3-phosphate dehydrogenase. 


\section{Fasudil induced ESCC cell apoptosis effectively}

We assessed the effect of fasudil on apoptosis in human oesophageal squamous cell carcinoma cells in vitro by DAPI staining. Figure $1 \mathrm{~B}$ shows that treatment with PBS (blank) did not obviously induce apoptosis in cells, but typical morphological alters associated with apoptosis-chromatin condensation, apoptotic body formation, and DNA fragmentation were prominently detected in fasudil-treated EC109 and KYSE150 cells. The addition of fasudil to cultured cells caused an obviously induction of apoptosis in a dose-dependent manner compared with the control cultures $(\mathrm{P}<0.001)$. Furthermore, procaspase- 3 cleaved to yield $17 / 19 \mathrm{kD}$ fragments, and procaspase-9 cleaved to produce a $35 / 37 \mathrm{kD}$ band that was also detected in EC109 and KYSE150 cells after fasudil treatment (Figure 1C). These results indicate that fasudil indeed induced apoptosis in cancer.

\section{Fasudil effectively induced ESCC cell autophagy}

Autophagy analysis was performed by immunoblotting for microtubule-associated protein 1B-light chain 3 (LC3), as previously described
[16-18]. Fasudil can induce the switch of LC3-I to LC3-II in a dose- and time-dependent manner in EC109 and KYSE150 cells (Figure 2A and 2B), showing that autophagy might be induced by fasudil. Consistently, P62, a regulator of the autophagic pathway, was obviously down-regulated in fasudil-treated cells. Nevertheless, Fasudil also induced an increase of Beclin-1 in ESCC cells similar to its effects on conventional autophagy.

\section{Inhibition of fasudil-induced autophagy enhances the apoptotic effect of fasudil}

Much research has shown that autophagy may act as a protective response in protecting tumour cells from therapy-induced cell apoptosis. We treated ESCC cells with fasudil in combination with a pharmacological autophagy inhibitor, chloroquine, that prevents autophagosome degradation. Compared with the treatment of fasudil or chloroquine alone, treatment of ESCC cells with fasudil and chloroquine led to an obvious inhibition of cell proliferation $(\mathrm{P}<0.001$, Figure. $3 \mathrm{~A}$ and $3 \mathrm{~B})$ and foci formation $(P<0.001$, Figure. $3 C)$ and an increase in the induction of apoptosis (Figure 3D).

A
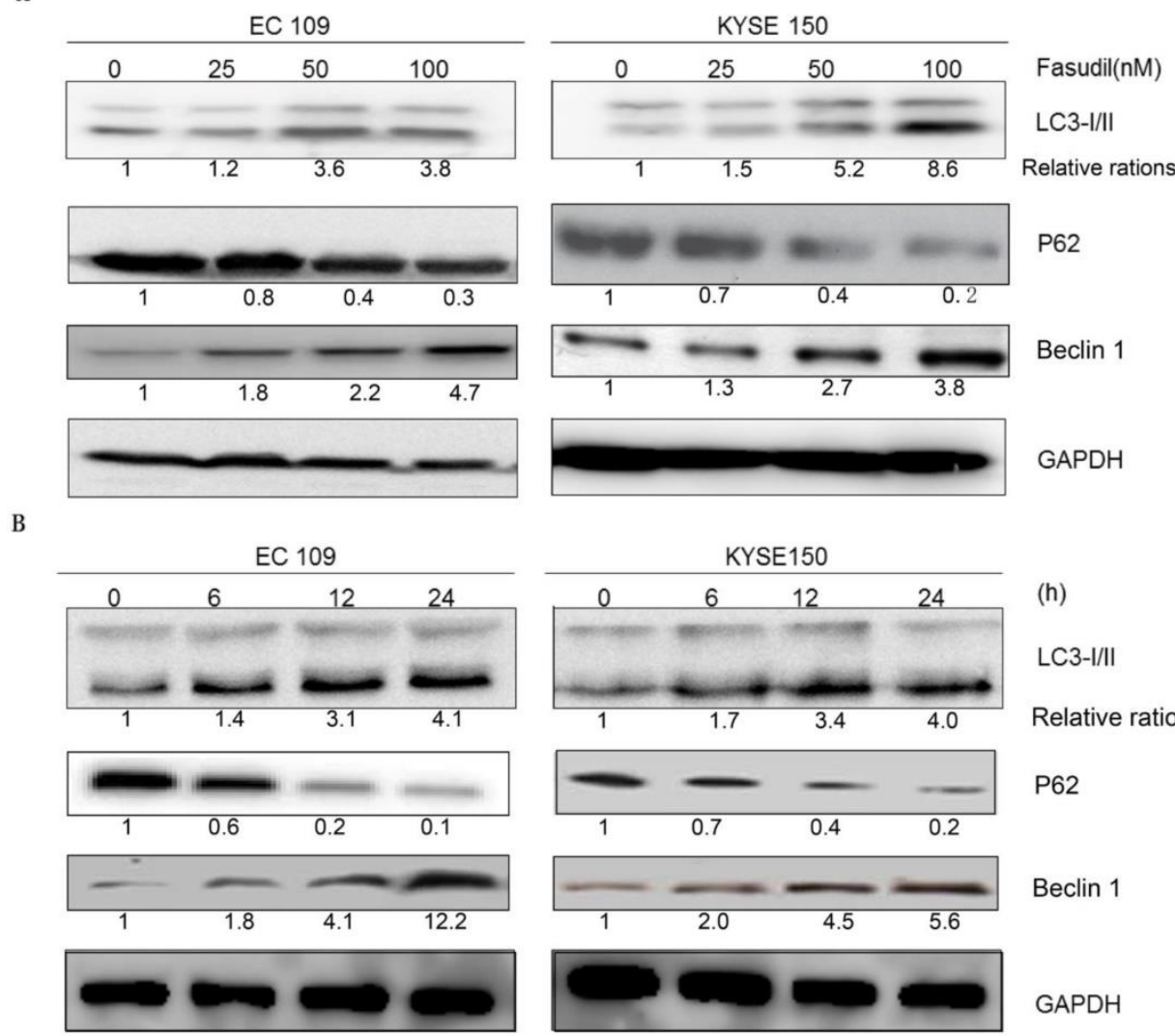

P62

Beclin 1

GAPDH

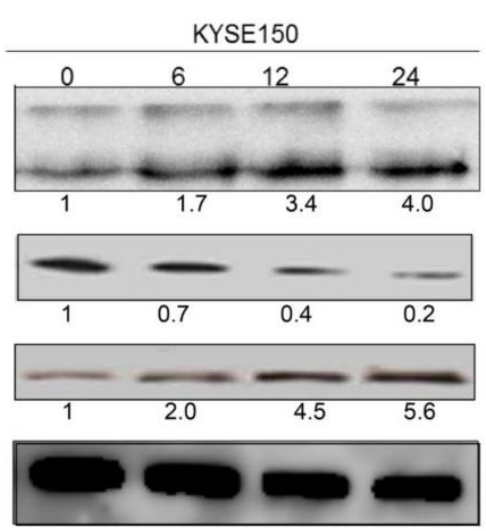

(h)

LC3-1/II

Relative rations

P62

Beclin 1

GAPDH

Figure 2. Fasudil induces autophagy in ESCC cells. (A) Western blot showing an increase in LC3- II and Beclin 1 levels in EC109 and KYSE150 cells after treatment with fasudil, while decrease in P62. The cells were treated with 0, 25, 50 or $100 \mathrm{nM}$ for $24 \mathrm{~h}$. (B) Western blot showing an increase in LC3-Il and Beclin 1 levels in cells treated with or without $50 \mathrm{nM}$ fasudil for the indicated time, decreases in P62. 
A
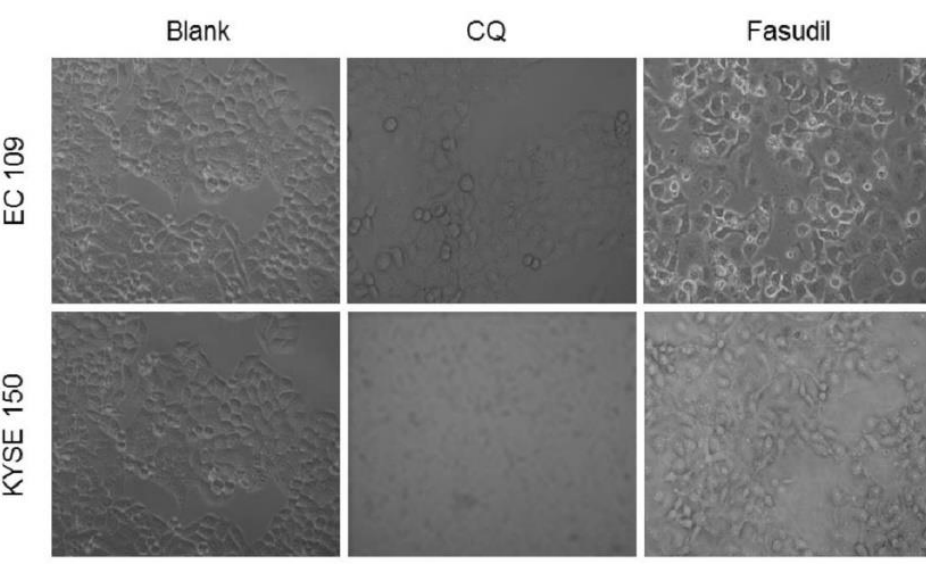

B
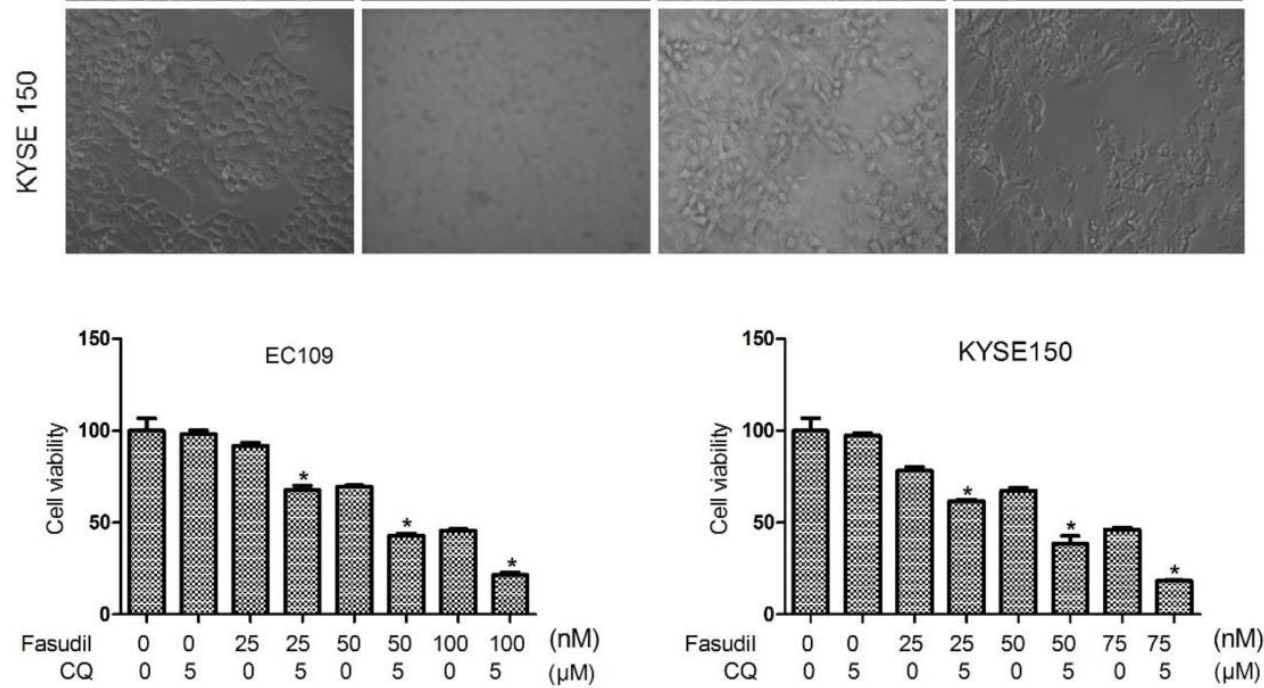

C

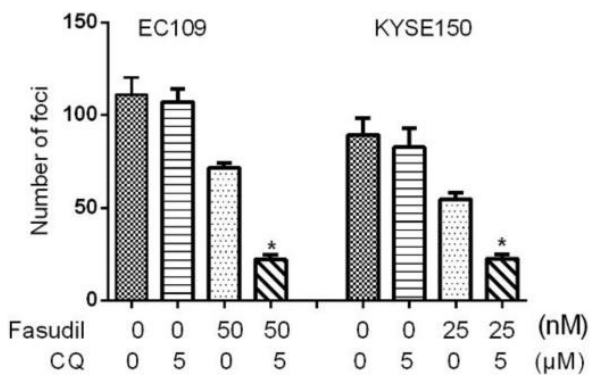

D

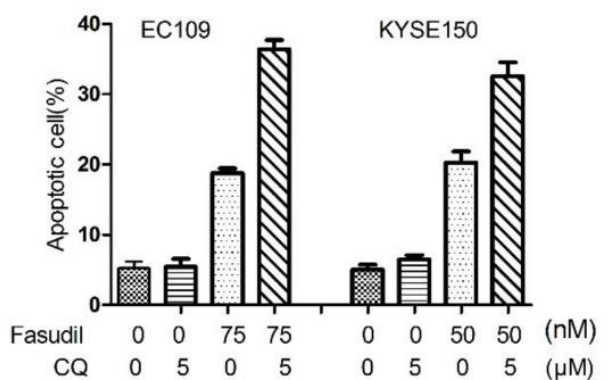

Figure 3. Chloroquine (CQ) promotes the apoptotic effect of fasudil in ESCC cells. (A) Marked morphological changes induced by fasudil or/and CQ in ESCC cells. EC109 and KYSE 150 cells were treated with medium alone, $75 \mathrm{nM}$ fasudil, $5 \mu \mathrm{M}$ CQ alone or their combination for $24 \mathrm{~h}$. Magnification is $\times 400$. (B) Cell growth assay showing that combined treatment with fasudil $(75 \mathrm{nM} \times 72 \mathrm{~h})$ and chloroquine $(5 \mu \mathrm{M} \times 72 \mathrm{~h})$ induces a lower growth rate in ESCC cells than treatment with fasudil only $(* \mathrm{P}<0.001$, fasudil plus chloroquine vs fasudil). (C) Representative inhibition of foci formation in culture by fasudil and/or chloroquine; quantitative analyses of foci numbers. Columns, mean of at least 3 independent assays; bars, SD. $P<0.001$, fasudil versus fasudil combined with chloroquine by Student's t-test. (D) Annexin V/PI staining assay showing that combined treatment with fasudil ( 75 $\mathrm{nM} \times 48 \mathrm{~h})$ and chloroquine $(5 \mu \mathrm{M} \times 48 \mathrm{~h})$ induces a higher rate of apoptosis than treatment with fasudil only $(* \mathrm{P}<0.05)$.

To further determine the protective role of autophagy, we utilized siRNA to silence the expression of Beclin 1 in ESCC cells (Figure. 4A). Compared with the results in siRNA controls, Beclin 1 knockdown impeded the fasudil-induced raise in LC3-II levels, indicating that autophagy is obviously suppressed by fasudil treatment (Figure. 4B). ATG7 knockdown also significantly enhanced fasudilinduced apoptosis (Figure. 4C). Caspase-3 and caspase-9 activity after fasudil treatment was clearly higher in fasudil-treated Beclin knockdown cells than in the fasudil-treated cells alone (Figure 4D and 4E). Similar to effect of Beclin 1 siRNA on fasudil-induced autophagy and apoptosis, ATG 7 siRNA also strongly impeded autophagy (Figure 4D) and increase apoptotic cells (Figure 4E), compared with siRNA controls. Our studies indicating that the inhibition of fasudil-induced autophagy enhances the potential for apoptosis after fasudil treatment.

\section{Discussion}

Autophagy is recognized as an evolutionarily conserved, intracellular self-defence mechanism responsible for degrading unnecessary or dysfunctional cellular organelles[19]. There is much proof to show that autophagy is a "double-edged sword" since it can be either protective or damaging, and the effects may change during tumour 
A

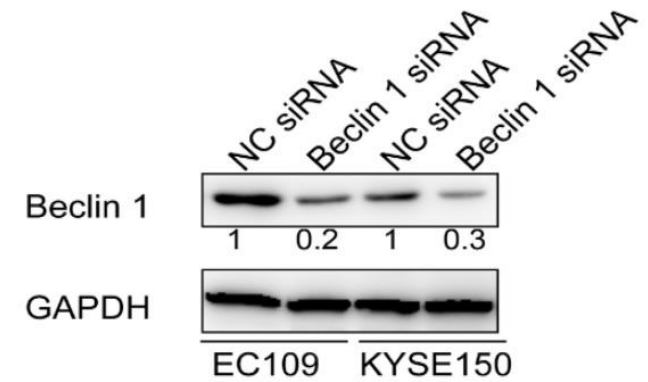

C

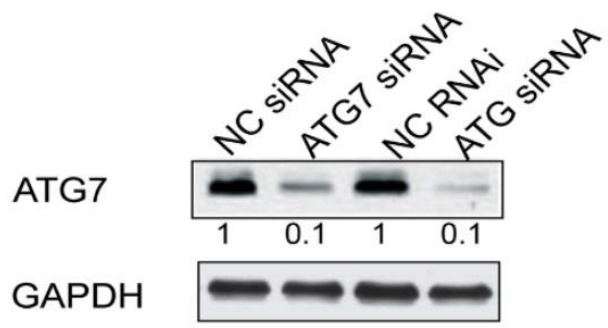

B

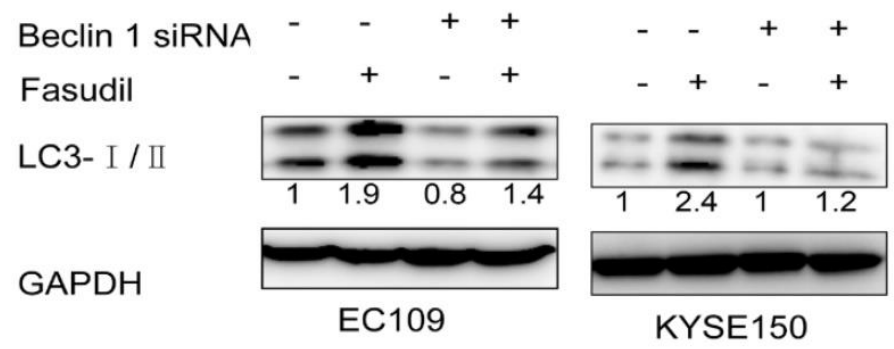

D

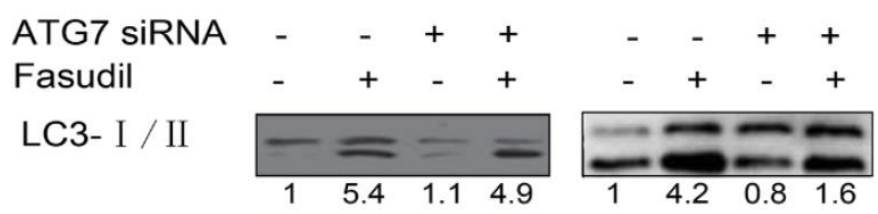

E
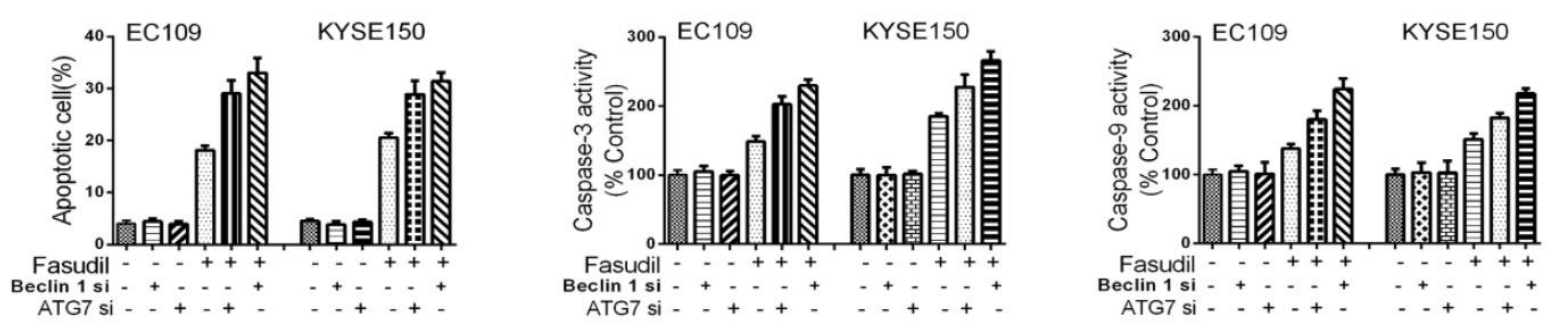

Figure 4. Effect of inhibition of Beclin 1 or ATG 7 expression on fasudil-induced apoptosis in ESCC cells. (A) Western blot showing a decrease in the expression of Beclin 1 (or ATG7, figure 4C) when EC109 and KYSE150 cells were transfected with Beclin 1 targeting siRNA vs negative control siRNA. (B) Western blot indicating that silence of Beclin 1 (or ATG 7, figure 4D) with siRNA impeded fasudil-induced raise in LC3-II levels in EC109 and KYSE150 cells. (E) Fasudil combined with autophagy inhibitor effectively induced ESCC cells apoptosis. Combination therapy with fasudil $(75 \mathrm{nM} \times 48 \mathrm{~h})$ and chloroquine $(5 \mathrm{mM} \times 48 \mathrm{~h})$ led to more ESCC cells apoptosis, higher caspase- 3 and caspase- 9 activities than fasudil only $(* \mathrm{P}<0.05$, fasudil plus Beclin 1 (or ATG 7) siRNA vs fasudil).

progression. In most studies, the inhibition of autophagy leads to increased oesophageal cancer cell apoptosis, indicating that autophagy might be a pro-survival mechanism rather than a cell death mechanism[20]. Herein, we show that the LC3-I to LC3-II conversion in fasudil-treated cells was increased in comparison with untreated cells. To date, no research has showed whether fasudil can induce autophagy in ESCC.

A large number of preclinical evidence shows that autophagy inhibition is currently being used as a novel anticancer therapeutic strategy, including for ESCC[21, 22]. Chloroquine (CQ) is well recognized as an autophagy inhibitor that controls acidic lysosomes. Our study showed that CQ combined with fasudil could significantly enhance anti-cancer effects by inducing apoptosis. To further detect the protective role of autophagy, we treated ESCC cells with Beclin 1 siRNA. Transfected ESCC cell lines were successfully established, and we verified that Beclin 1 siRNA reduced Beclin 1 protein expression by western blot. Using flow cytometry analysis, it was subsequently demonstrated that compared with the control siRNA group, the knockdown of Beclin 1 in ESCC cells significantly induced apoptosis with fasudil treatment. A caspase activity assay was then performed to examine the activation of caspases as a possible mechanism underlying the increased levels of apoptosis observed in Beclin 1 knockdown ESCC cells. A significant increase in active caspase- 3 and caspase-9 were observed in Beclin 1 siRNAtransfected ESCC cells. This was consistent with the results obtained by FACS analysis. Similar to effect of Beclin 1 siRNA on fasudil-induced autophagy and apoptosis, Atg7 siRNA also strongly prevented autophagy and increased apoptotic cells compared with siRNA controls. Therefore, autophagy inhibition induced strong activation of caspase-3 and caspase-9 in ESCC cells, which suggests that caspase-dependent apoptosis may have contributed to the synergistic 
anticancer effects of fasudil combined with an autophagy inhibitor observed in ESCC cells.

In summary, we have shown that the RhoA inhibitor fasudil has an anti-cancer effect in ESCC; it may induce ESCC cell apoptosis and autophagy. In addition, our findings suggest a novel approach to improve fasudil anti-cancer effects related to autophagy inhibition. This combined therapy increases the level of apoptosis in ESCC cells, resulting in effective growth inhibition and elimination of cancer cells. Clinical research on targeting autophagy provides a promising therapeutic strategy to overcome resistance and enhance the effects of anticancer therapies in cancer patients[17].

\section{Abbreviations}

ESCC: Oesophageal squamous cell carcinoma; CQ: Chloroquine.

\section{Acknowledgements}

We thank Professor Hong-Chuan Jin, Doctor Hai-Qi Lu and Doctor Fa-Liang Wang from the Laboratory of Cancer Biology, Sir Runrun Shaw Hospital, Medical School of Zhejiang University, for their excellent advice and support technical assistance.

\section{Funding}

This work was supported in part by the Zhejiang Provincial Medical and Health Research Plan (2018262060, 201482897, 2017194174 and 2015119812); the China Postdoctoral Science Foundation (2012 M521189); and the Zhejiang Province Postdoctoral Science Foundation for Excellent Post-doctors (Bsh1202064).

\section{Ethical approval}

This article does not contain any studies with human participants or animals performed by any of the authors.

\section{Competing Interests}

The authors have declared that no competing interest exists.

\section{References}

1. Pennathur A, Gibson MK, Jobe BA, Luketich JD. Oesophageal carcinoma. Lancet. 2013; 381: 400-12.

2. Katlic MR, Wilkins EW, Jr., Grillo HC. Three decades of treatment of esophageal squamous carcinoma at the Massachusetts General Hospital. The Journal of thoracic and cardiovascular surgery. 1990; 99: 929-38.

3. Wang $\mathrm{K}, \mathrm{Ma} \mathrm{W}$, Wang J, Yu L, Zhang X, Wang Z, et al. Tumor-stroma ratio is an independent predictor for survival in esophageal squamous cell carcinoma. Journal of thoracic oncology: official publication of the International Association for the Study of Lung Cancer. 2012; 7: 1457-61.

4. Faried A, Faried LS, Kimura H, Nakajima M, Sohda M, Miyazaki T, et al. RhoA and RhoC proteins promote both cell proliferation and cell invasion of human oesophageal squamous cell carcinoma cell lines in vitro and in vivo. European journal of cancer. 2006; 42: 1455-65.
5. Faried A, Nakajima M, Sohda M, Miyazaki T, Kato H, Kuwano H. Correlation between RhoA overexpression and tumour progression in esophageal squamous cell carcinoma. European journal of surgical oncology: the journal of the European Society of Surgical Oncology and the British Association of Surgical Oncology. 2005; 31: 410-4.

6. Gurkar AU, Chu K, Raj L, Bouley R, Lee SH, Kim YB, et al. Identification of ROCK1 kinase as a critical regulator of Beclin1-mediated autophagy during metabolic stress. Nat Commun. 2013; 4: 2189.

7. Ma J, Zhang J, Ma Y, Zheng J, Cheng Y, Xue Y, et al. Adenovirus-mediated RhoA shRNA suppresses growth of esophageal squamous cell carcinoma cells in vitro and in vivo. Medical oncology. 2012; 29: 119-26.

8. Russell SJ, Peng KW, Bell JC. Oncolytic virotherapy. Nature biotechnology. 2012; 30: 658-70.

9. Satoh SI, Ikegaki I, Kawasaki K, Asano T, Shibuya M. Pleiotropic Effects of the Rho-kinase Inhibitor Fasudil After Subarachnoid Hemorrhage: A Review of Preclinical and Clinical Studies. Current vascular pharmacology. 2014.

10. Miyamoto C, Maehata Y, Ozawa S, Ikoma T, Kubota E, Izukuri K, et al. Fasudil suppresses fibrosarcoma growth by stimulating secretion of the chemokine CXCL14/BRAK. Journal of pharmacological sciences. 2012; 120: 241-9.

11. Takeba Y, Matsumoto N, Watanabe M, Takenoshita-Nakaya S, Ohta Y, Kumai $\mathrm{T}$, et al. The Rho kinase inhibitor fasudil is involved in p53-mediated apoptosis in human hepatocellular carcinoma cells. Cancer chemotherapy and pharmacology. 2012; 69: 1545-55.

12. Wermke M, Camgoz A, Paszkowski-Rogacz M, Thieme S, von Bonin M, Dahl A, et al. RNAi profiling of primary human AML cells identifies ROCK1 as a therapeutic target and nominates fasudil as an antileukemic drug. Blood. 2015; 125: 3760-8.

13. Yang $X$, Liu $Y$, Zong Z, Tian D. The Rho kinase inhibitor fasudil inhibits the migratory behaviour of 95-D lung carcinoma cells. Biomedicine \& pharmacotherapy = Biomedecine \& pharmacotherapie. 2010; 64: 58-62.

14. Takata K, Morishige $\mathrm{K}$, Takahashi T, Hashimoto K, Tsutsumi S, Yin L, et al. Fasudil-induced hypoxia-inducible factor-1alpha degradation disrupts a hypoxia-driven vascular endothelial growth factor autocrine mechanism in endothelial cells. Molecular cancer therapeutics. 2008; 7: 1551-61.

15. Xie F, Liu H, Zhu YH, Oin YR, Dai Y, Zeng T, et al. Overexpression of GPR39 contributes to malignant development of human esophageal squamous cell carcinoma. BMC Cancer. 2011; 11: 86.

16. Shen J, Zheng H, Ruan J, Fang W, Li A, Tian G, et al. Autophagy inhibition induces enhanced proapoptotic effects of ZD6474 in glioblastoma. Br J Cancer. 2013; 109: 164-71.

17. Lu Z, Xu N, He B, Pan C, Lan Y, Zhou H, et al. Inhibition of autophagy enhances the selective anti-cancer activity of tigecycline to overcome drug resistance in the treatment of chronic myeloid leukemia. J Exp Clin Cancer Res. 2017; 36: 43

18. Xu XD, Zhao Y, Zhang M, He RZ, Shi XH, Guo XJ, et al. Inhibition of Autophagy by Deguelin Sensitizes Pancreatic Cancer Cells to Doxorubicin. Int J Mol Sci. 2017; 18.

19. Di Benedetto G, Gerbino A, Lefkimmiatis K. Shaping mitochondrial dynamics: The role of cAMP signalling. Biochem Biophys Res Commun. 2017.

20. Zhu X, Pan Q, Huang N, Wu J, Zhen N, Sun F, et al. RAD51 regulates CHK1 stability via autophagy to promote cell growth in esophageal squamous carcinoma cells. Tumour Biol. 2016.

21. Lu C, Xie C. Radiation-induced autophagy promotes esophageal squamous cell carcinoma cell survival via the LKB1 pathway. Oncol Rep. 2016; 35: 3559-65.

22. Yoshida GJ. Therapeutic strategies of drug repositioning targeting autophagy to induce cancer cell death: from pathophysiology to treatment. J Hematol Oncol. 2017; 10: 67. 